\title{
Deleterious CHEK2 Gene Mutation
}

National Cancer Institute

\section{Source}

National Cancer Institute. Deleterious CHEK2 Gene Mutation. NCI Thesaurus. Code

C134511.

A change in the nucleotide sequence of the CHEK2 gene that is associated with increased risk of disease. 